\title{
Canopy openness and soil conditions explain community structure and diversity in a tropical seasonal forest in south-eastern Brazil
}

\author{
Tiago Egydio Barreto ${ }^{1}$, Natália Macedo Ivanauskas ${ }^{2 *}$ (D), Renato Augusto Ferreira de Lima ${ }^{3}$ (D), \\ Maria Teresa Zugliani Toniato ${ }^{2}$ (D), Flaviana Maluf Souza ${ }^{2}$ (1) and Ricardo Ribeiro Rodrigues ${ }^{4}$ (1)
}

Received: June 16, 2020

Accepted: April 16, 2021

\begin{abstract}
In tropical semi-deciduous forests, where $20-50 \%$ of canopy trees shed their leaves in the dry season, species with varying degrees of leaf deciduousness share the same space and resources. Here, we describe the tree community in a 10.24-ha plot to assess whether small-scale variation in canopy structure and soil conditions are associated with changes in tree community structure, diversity, and composition. We sampled 11,585 individuals with diameter at breast height $\geq 4.8 \mathrm{~cm}$ belonging to 146 species. Plot density $\left(1,129\right.$ trees ha-1) and basal area $\left(24.81 \mathrm{~m}^{2} \mathrm{ha} \mathrm{a}^{-1}\right)$ were smaller than other similar forests, which may be due to an old wind disturbance. For 8.96 ha, we evaluated the relationship between abiotic factors and community descriptors using regression models. Results varied within size classes, but canopy openness was associated with changes in the community structure and diversity, and soil fertility did not affect species diversity. Tree density, basal area, and diversity were smaller in areas with more canopy gaps. Tree density and basal area increase with phosphorus availability, while the density of deciduous trees increased with canopy openness and base saturation. Thus, we found evidence that canopy openness and soil can explain small-scale variations of forest structure and diversity.
\end{abstract}

Keywords: Atlantic Forest, environmental heterogeneity, permanent plot, regression models, species richness

\section{Introduction}

Tropical rain forests are dominated by evergreen tree species in regions with no pronounced dry season. However, there are vast tropical and subtropical areas with a marked dry season that are covered by seasonal (semi-deciduous and deciduous) forests (Givnish 2002; Oliveira-Filho et al. 2006). Semi-deciduous forests are characterized by the deciduousness of up to $50 \%$ of their trees during the dry season (IBGE 2012). In these forests, one key question is how evergreen, deciduous, and semi-deciduous species can co- occur in the same stand and what abiotic factors determine their dominance (Walters \& Reich 1999; Namikawa et al. 2000; Niinemets 2010; Pérez-Harguindeguy et al. 2013). Evergreen species should be favored by a longer growing season (i.e., greater the leaf longevity) and a higher leaf allocation ratio in comparison to deciduous trees. On the other hand, deciduous trees have higher photosynthetic rates and hydraulic conductivities (Sobrado 1993; Vico et al. 2017).

The combination of rainfall seasonality and canopy deciduousness imposes to species of semi-deciduous forests

\footnotetext{
1 Programa de Pós-Graduação em Biologia Vegetal, Instituto de Biologia Universidade Estadual de Campinas, 13083-000, Campinas, SP, Brazil 2 Instituto de Pesquisas Ambientais, 02378-000, São Paulo, SP, Brazil

3 Departamento de Ecologia, Instituto de Biociências, Universidade de São Paulo, 05508-900, São Paulo, SP, Brazil

4 Departamento de Ciências Biológicas, Escola Superior de Agricultura “Luiz de Queiroz”, Universidade de São Paulo, 13418-900, Piracicaba, SP, Brazil
}

* Corresponding author: nivanaus@yahoo.com.br 
the necessity to cope with both drought and higher light incidence to survive (Gandolfi et al. 2009). However, variation in soil conditions across the forest (e.g., soil depth and water availability) can influence species responses to increased drought and light incidence. For instance, greater soil infertility, rooting depth and permeability can favor the evergreen habit (Givnish 2002). Therefore, smallscale variation in soil conditions can promote resource heterogeneity and thus generate differences in plant composition, phenology, structure and ultimately contribute to the maintenance of the high species diversity in tropical forests (Molino \& Sabatier 2001).

Besides soil conditions, disturbances can also play an important role in generating resource heterogeneity at small spatial scales. Canopy gaps caused by the fall of trees or their branches typically generate changes in both light intensity and quality (Lima \& Gandolfi 2009). Multiple aspects of forest succession are related to canopy gap dynamics, which directly affects the diversity and total density of understory plant individuals (Brenes-Arguedas et al. 2011; Halpern \& Lutz 2013). The differential performance of shade tolerant and intolerant species under distinct light regimes has been considered one of the most important factors to explain tree species distribution in tropical forests (Denslow 1980; Whitmore 1989; Whitmore 1996). In addition, the vertical competition for light resources among tree crowns can promote the coexistence of species (Kohyama 1993). Therefore, the vertical heterogeneity in light resources (reflected in the size distribution of trees) as well as the horizontal heterogeneity (reflected by the canopy gap dynamics) play key roles on forest organization.

However, few studies have evaluated the effect of canopy openness and the associated changes in light regimes in semi-deciduous forests, with the exception being Gandolfi et al. (2007; 2009), who introduced the concept of "gaps of deciduousness" for these forests. Gaps of deciduousness are seasonally recurring opening in the canopy caused by leaf fall, a cyclical phenomenon completely different from treefall gaps. In seasonal forests, gaps of deciduousness coincide with the dry season, when there is a reduction in soil water availability. This results in a selective scenario favorable for seedlings that can grow under low water availability and elevated light incidence. Each canopy tree may create specific microsite conditions below its crown, which works as a filter for those species that attempt to regenerate below it (Gandolfi et al. 2007). Photosynthesis by an evergreen tree during the dry season should be greater if it is surrounded or coverage by deciduous trees, and lower if it is instead surrounded or coverage by other evergreens (Givnish 2002).

Here we investigate how small-scale environmental heterogeneity in understory light regimes caused by canopy openings and soil properties can influence tree community structure and diversity in a semi-deciduous Atlantic Forest, south-eastern Brazil. Thus, we first (1) provide a general description of the plot tree community and compare the main patterns found to similar forests. Next, we evaluate (2) whether soil nutrients (V \%, OM, P and the S-index) and/or canopy gap area (used here as an indirect measure of the understory light conditions) are associated with small-scale changes in the structure and diversity of the community. Finally, we assess (3) whether sites with lower soil water retention capacity and shallow rooting systems would be more associated to the higher concentration of deciduous trees.

\section{Materials and methods}

\section{Study area}

The Caetetus Ecological Station (2,179-ha) is a protected area that belongs to the Gália and Alvinlândia Counties, São Paulo State, Southeastern Brazil (Fig. 1). It is the second largest remnant in the southwest region of the State of São Paulo (Ramos et al. 2008). Elevation ranges from 500 to 680 meters above sea level and climate is classified as Cwa (Köppen 1948), which is subtropical humid climate with a marked dry season during winter. The average annual temperature is $21^{\circ} \mathrm{C}$, with monthly average temperatures ranging between $17^{\circ} \mathrm{C}$ and $25^{\circ} \mathrm{C}$. Average annual precipitation is around $1,303 \mathrm{~mm}$, with a dry season between April and September (monthly precipitation < 100 $\mathrm{mm}$ ). Because of the interannual climatic variability, the dry season can be longer in some years than others, increasing water shortage beyond normal indexes.

The vegetation is classified as a Montane Semi-deciduous Seasonal Forest (IBGE 2012). Inside the permanent forest plot studied here, three soil types were classified according to Soil Taxonomy (Soil Survey Staff 2014): Arenic Haplustult, Arenic Haplustalf and Aquertic Haplustalf. The Arenic Haplustult and Arenic Haplustalf dominate and occur on hilly terrain, while the Aquertic Haplustalfs are associated with the floodplain of a first-order channel, located in the north-western side of the plot. Detailed information regarding the morphological properties of the horizons and the hydro-physical characterization of the soils are described in Cooper et al. (2012).

\section{Tree census}

The permanent forest plot has 10.24 ha $(320 \times 320 \mathrm{~m})$ and is subdivided into 256 subplots of $20 \times 20 \mathrm{~m}$. The plot was established in 2002 in the central part of the Caetetus Ecological Station ( $49^{\circ} 42^{\prime} 04.13^{\prime \prime}$ W and $22^{\circ} 40^{\prime} 40.45^{\prime \prime}$ S). The plot was first censused in 2002/2003, then in 2004/2005 and in 2010. The data presented here refer to the individuals found alive during the 2010 census. Tree census followed the Center for Tropical Forest Science protocol (Condit 1998) except that it only included individuals with stem girth at breast height $\geq 15 \mathrm{~cm}$, equivalent to a Diameter at Breast Height $(\mathrm{DBH})$ of $\geq 4.8 \mathrm{~cm}$. All individuals were 
Tiago Egydio Barreto, Natália Macedo Ivanauskas, Renato Augusto Ferreira de Lima, Maria Teresa Zugliani Toniato, Flaviana Maluf Souza and Ricardo Ribeiro Rodrigues
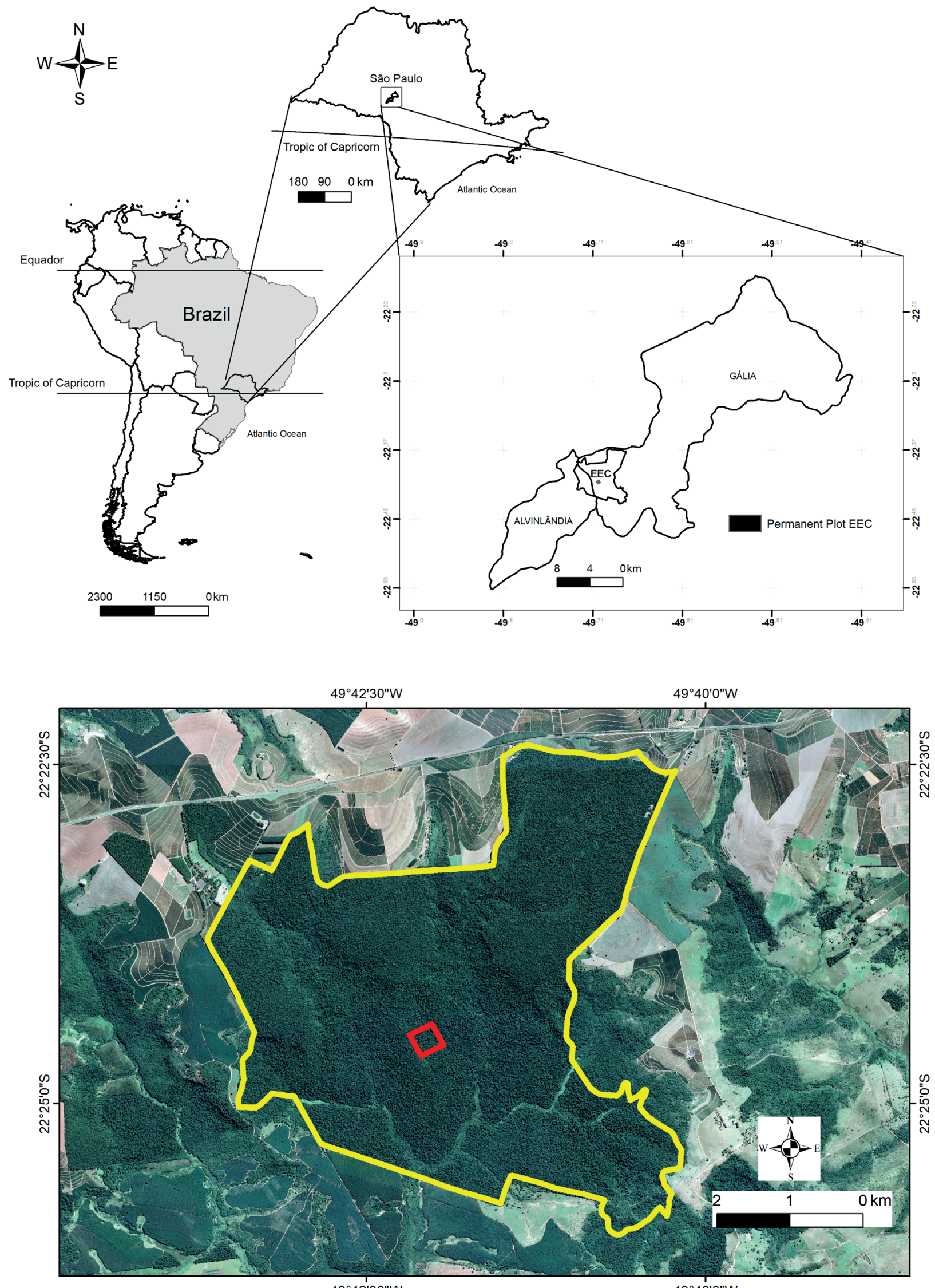

Figure 1. Location of the Caetetus Ecological Station and the 10.24-ha forest permanent plot (red square). Source: Marco Nalon 


\section{Canopy openness and soil conditions explain community structure and diversity in a tropical seasonal forest in south-eastern Brazil}

tagged, mapped, measured, and identified to the species level. The diameter of each stem was measured to the nearest millimeter and, for multi-stemmed individuals (i.e., with ramifications of the trunk below $1.3 \mathrm{~m}$ ), the individual was included when at least one of the branches obeyed the inclusion criterion, and the DBHs of all branches were then recorded for the calculation of the basal area (the sum of the cross-sectional areas of the multiple stems).

Species identifications were based on the comparison with materials deposited in ESA and SPSF herbaria and on the consultation of specialists and the specialized literature (e.g., Ramos et al. 2008) and voucher of the species were compiled by Cunha (2016). Spelling and synonyms followed Flora do Brasil 2020 (2018).

\section{Leaf phenological patterns}

The trees of each species were grouped into three categories of leafing pattern: evergreen, semi-deciduous and deciduous. We categorized the tree species using the observations of phenophases in the field and by information from the literature (Morellato et al. 1989).

\section{Forest structure and diversity}

The general description of the tree community was performed for the entire 10.24-ha permanent plot. In order to assess possible differences in the vertical stratification of the forest, we provide a description for all trees sampled in the plot $(\mathrm{DBH} \geq 4.8 \mathrm{~cm})$ and descriptions for different size classes: $4.8 \leq \mathrm{DBH}<10,10 \leq \mathrm{DBH}<20,20 \leq \mathrm{DBH}$ $<30$ and DBH $\geq 30 \mathrm{~cm}$. For multi-stemmed individuals, the diameter of the largest stem was used to determine the size class. Although there are variations in the heightDBH relationship among tree species, we assume that they are closely related; we thus used $\mathrm{DBH}$ as a proxy of tree positioning in respect to the forest vertical stratification (Scaranello et al. 2012; Mugasha et al. 2013).

For each size class, we calculated total tree density, basal area, richness, singletons, evenness $(\mathrm{J})$, Shannon $\left(\mathrm{H}^{\prime}\right)$, Fisher's alpha and Berger-parker index (Mueller-Dombois \& Ellenberg 1974; Magurran 2011). We used different indices to measure species diversity, which are slightly different in how they express diversity (Melo 2008), because some indices take more into account the density of the most dominant species in the sample (e.g., Berger-Parker, Simpson), while other indices weigh more the rare species (e.g., singletons and Fisher's alpha). In addition, we report different diversity indices to increase the comparability of our results with a wider range of studies using different types of indices. We also estimated the average value per hectare for the same descriptors of forest structure and diversity. This procedure was performed by sampling at random and without replacement 1,000 draws of $2520 \times 20$ m subplots (total of 1 ha) from the total of 256 subplots. We used the statistical program R (R Development Core Team 2014).
We estimated the richness using different non-parametric estimators (e.g., Michaelis-Menten, first and second order Jackknife), performed using 1,000 randomizations using the EstimateS software (Colwell 2006). The choice of using the first order Jackknife was made empirically, based on the number of samples required to estimate the total plot richness and on the tendency of stabilization of species accumulation curve (not shown), following Colwell and Coddington's (1994) recommendations. The number of singletons per hectare was used here as a practical definition of rare species. Fisher's alpha $\left[S=\alpha \ln \left(1+N . \alpha^{-1}\right)\right]$ describes the relationship between the number of species $(S)$ and the number of individuals $(\mathrm{N})$ in a community and is less affected by sample size and the abundance of common species. The Berger-parker index refers to the relative abundance of the most common species.

\section{Canopy gap area}

In $2005,8.96$ ha (224 contiguous subplots of $20 \times 20$ $\mathrm{m}$ or $280 \times 320 \mathrm{~m}$ ) of the permanent plot was surveyed for canopy gaps during the dry season (Lima et al. 2008). Gaps were defined as the absence from the canopy of at least one-half of a tree (Runkle 1982). Gap delimitation method followed Runkle (1982), i.e., 'the ground area under a canopy opening extending to the bases of canopy trees surrounding the canopy opening'. A minimum size of 20 $\mathrm{cm}$ DBH was used to define the surrounding canopy trees. Canopy gap size was measured using the method proposed by Lima (2005), which consists in dividing the gap area into triangles, measuring the side of each triangle, and then summing their area. Large gaps exceeding the plot limits were not completely measured (only the gap area inside the plot was measured). We calculated the gap size area inside each $20 \times 20 \mathrm{~m}$ subplot, and we assigned a zero value for the subplots without any gaps. No distinction between single and complex gaps (i.e., gaps formed by distinct episodes of tree mortality) was made to calculate gap area inside the subplots. The total area of gaps was of 3.20 ha ( $36 \%$ of the 8.96-ha mapped area).

\section{Edaphic properties}

Soil samples were collected at three deeps $(0-5 ; 10-20$; $80-100 \mathrm{~cm}$ ) in the center of all 256 subplots (Vidal-Torrado et al. 2021). The chemical analyses were performed according to the Soil Analysis Manual Methods of the Brazilian Agricultural Research Corporation (EMBRAPA 1997). We used base saturation (V \%), organic matter content $\left(\mathrm{OM}-\mathrm{g} \mathrm{dm}^{-3}\right)$ and phosphorus $\left(\mathrm{P}-\mathrm{mg} \mathrm{kg}^{-1}\right)$ as soil fertility descriptors. The $\mathrm{S}$-index was used as an indicator of soil physical qualities since it is associated with soil rootability and water availability to plants. The $S$ value is defined as the absolute value of the slope of water retention curve at its inflection point (Dexter 2004). It is indicative of the extent to which the soil porosity is concentrated into a narrow range of pore sizes, being correlated to bulk density and 
total porosity (van Lier 2014). S values greater than 0.03 do not restrict root growth; reduction of root growth occurs between 0.02 and 0.03 ; and root penetration is impeded below 0.02 . We used the $S$ index from samples collected in the deeper layer $(80-100 \mathrm{~cm})$ of the most representative soil types of the permanent plot (Cooper et al. 2012), which ranged from 0.01 to 0.09 among subplots.

\section{Environmental heterogeneity}

We constructed regression models to describe the relationship between the abiotic factors (explanatory variables) and the tree community descriptors (response variables). These models were constructed using the data available for the 8.96-ha plot for which we had all variables available (the $280 \times 320 \mathrm{~m}$ area that was surveyed for canopy gaps). Models were constructed for the $\geq 4.8$ and $\geq 20 \mathrm{~cm}$ DBH size classes, in order to assess possible differences in the results including or excluding individuals in the forest understory. We assumed that overstory trees may influence the structure of the understory (Souza et al. 2015), with light entrance and soil nutrients depending on the leaf phenology and disturbances of canopy trees (branch-falls or death of part or whole tree).

We used tree density (n) and basal area $\left(\mathrm{m}^{2}\right)$ in each $20 \times 20 \mathrm{~m}$ subplot to describe the forest structure. Regarding the forest composition, we used the relative density of evergreen, semi-deciduous and deciduous trees (relative density: proportion of individuals in each category per subplot). For diversity, we used the number of species per subplot (but controlling for the number of individuals sampled) and the Simpson and Fisher alpha diversity indexes, both calculated using the vegan package in the $\mathrm{R}$ statistical program (Oksanen et al. 2012). The explanatory variables of the regression models were the percentage of canopy gap area (used here as an indirect measure of the understory light conditions) and physical and chemical soil properties (P, OM, $\mathrm{V} \%$ and the S-index) for each $20 \times 20 \mathrm{~m}$ subplot.

Three regression models were constructed for each dependent variable $\left.\left(\mathrm{X}_{1}, \mathrm{X}_{2}, \ldots\right): 1\right)$ a null model (model without light and soil independent variables); 2) a model with light regime effect (canopy gap area per subplot as independent variable); and 3) a model with soil effect (soilrelated measurements as independent variables). Therefore, the following experimental statistical model was proposed: $Y_{i}=\beta_{0}+\beta_{1} X_{i 1}+\beta_{2} X_{i 2}+\varepsilon_{i}$.

Regression models included linear or generalized linear models (Pinheiro \& Bates 2000; Zuur et al. 2009). The decision between these classes of models was based on the best error distribution (e.g., Normal, Gaussian, Poisson, negative binomial) for each response variables. The regression models were inspected to make sure they meet the assumptions of normality of the residuals and to avoid collinearity between dependent variables (Bolker 2009). We also verified all models for possible heteroscedasticity and/ or spatial autocorrelation among observations. When there was evidence of spatial correlation between observations, we used spatial regression models and an exponential correlation structure (Pinheiro \& Bates 2000).

We initially tested soil type as a random variable in our models, but for none of the response variables there was an improve in model fit to data. We constructed and validated the models following the approach suggested by Zuur et al. (2009), which assists the selection of optimal model structure for inference. The assessment of the effect of canopy openness and soil effects on each response variable was based on standardized $t$ tests applied to the regression parameters. The Akaike's Information Criterion (AIC) was used to select the best models for each variable. We considered differences in AIC values greater than log (8) as indicators of different fits between models (Burnham \& Anderson 2002).

All statistical analyses were run using the 'vegan' (Oksanen et al. 2012), 'nlme' (Pinheiro et al. 2008), 'bblme' (Bolker 2009) and 'pscl' (Zeileis et al. 2008) packages in the R statistical program (R Development Core Team 2014).

\section{Results}

General description of forest structure and diversity

The 10-ha permanent plot contained more than 11,000 individuals with $\mathrm{DBH} \geq 4.8 \mathrm{~cm}$ (Tab. 1), belonging to 42 families, 106 genera and 146 species (Tab. 2). The plot is wellrepresented by some typical seasonal forests species that are globally endangered, such as Aspidosperma polyneuron and Balfourodendron riedelianum (IUCN 2018).

Despite of our sampling efforts (10.24 ha), the number of singletons for the entire plot did not differ from the estimated number of singletons per ha (Tab. 1), suggesting great species turnover in the community. With the increase of the DBH cutoff criteria, there was an expected decrease in the number of individuals and species sampled. However, there was an increase in equability and diversity in the upper strata, mostly due to the steep decline in the relative contribution of Metrodorea nigra, which often does not reach DBH over $10 \mathrm{~cm}$ (Tab. 1).

The most common species in the canopy layer (DBH $\geq 20 \mathrm{~cm}$ ) were Ocotea prolifera, Aspidosperma polyneuron, Centrolobium tomentosum, Croton floribundus and Balfourodendron riedelianum (Tab. 3). The midstory and understory layers $(\mathrm{DBH}<20 \mathrm{~cm}$ ) were dominated notably by Metrodorea nigra, Trichilia clausseni and Trichilia catigua, with $M$. nigra representing almost $40 \%$ of all individuals in the plot (Tab. 2). This species had densities way above all other populations in all strata below DBH $20 \mathrm{~cm}$ (Tab. 3, $\mathrm{DBH} \geq 4.8,4.8 \leq \mathrm{DBH}<10$ e $10 \leq \mathrm{DBH}<20$ ).

Another important parameter in the forest structure analysis in Caetetus was the basal area (Tab. 1). The high values of basal area in the first two diametric classes (DBH $\geq 4.8$ and $4.8 \leq \mathrm{DBH}<10$ ) are directly related to the number 


\section{Canopy openness and soil conditions explain community structure and diversity in a tropical seasonal forest in south-eastern Brazil}

Table 1. Total and per hectare values of the main structure and diversity parameters in different size classes in a 10.24-ha plot of tropical semi-deciduous forest in the Caetetus Ecological Station. Brackets refer to the 2.5 and 97.5 percentiles of the distribution obtained from 144 one-hectare plots inside the 10.24-ha plot. DBH = Diameter at Breast Height.

\begin{tabular}{|c|c|c|c|c|c|}
\hline \multirow{2}{*}{ Parameter } & \multicolumn{5}{|c|}{ Size class (cm) } \\
\hline & $\mathrm{DBH} \geq 4.8$ & $4.8 \leq \mathrm{DBH}<10$ & $10 \leq \mathrm{DBH}<20$ & $20 \leq \mathrm{DBH}<30$ & $\mathrm{DBH} \geq 30$ \\
\hline Density (n) & 11585 & 6408 & 3496 & 1012 & 669 \\
\hline Density per hectare (n. ha $\left.{ }^{-1}\right)$ & $1129(1052-1212)$ & $627(580-690)$ & $344(330-357)$ & $100(91-107)$ & $65(51-82)$ \\
\hline Total basal area $\left(\mathrm{m}^{2}\right)$ & 253.6 & 25.2 & 54.8 & 46.0 & 127.7 \\
\hline Área basal $\left(\mathrm{m}^{2} \cdot \mathrm{ha}^{-1}\right)$ & $24.8(20.8-29.7)$ & $2.6(2.3-2.8)$ & $5.4(4.9-5.9)$ & $4.7(4.1-5.3)$ & $12.8(9.5-16.5)$ \\
\hline Species (n) & 146 & 123 & 108 & 68 & 67 \\
\hline Species per hectare $\left(\mathrm{ha}^{-1}\right)$ & $82(72-92)$ & $25(47-63)$ & $52(44[59)$ & $29(23-34)$ & $26(20-32)$ \\
\hline Singletons (n) & 28 & 30 & 20 & 16 & 21 \\
\hline Singletons per hectare (n. ha ${ }^{-1}$ ) & $26(22-31)$ & $23(18-28)$ & $20(17-24)$ & $14(12-17)$ & $13(10-17)$ \\
\hline Shanon $\left(\mathrm{H}^{\prime}\right)$ & 2.90 & 2.34 & 3.05 & 3.18 & 3.30 \\
\hline Shanon per hectare $\left(\mathrm{ha}^{-1}\right)$ & $2.84 \pm 0.08$ & $2.20 \pm 0.12$ & $2.85 \pm 0.09$ & $2.83 \pm 0.13$ & $2.78 \pm 0.16$ \\
\hline Equability (J) & 0.58 & 0.49 & 0.65 & 0.75 & 0.79 \\
\hline Equability per hectare & $0.64(0.60-0.70)$ & $0.55(0.50-0.65)$ & $0.73(0.69-0.77)$ & $0.86(0.80-0.90)$ & $0.87(0.80-0.93)$ \\
\hline Fisher's alpha per hectare & $18.2(15.4-23.7)$ & $12.8(9.3-18.3)$ & $15.6(11.7-20.9)$ & $13.7(8.9-18.8)$ & $14.3(9.6-18.8)$ \\
\hline Berger-Parker per hectare & $0.4(0.3-0.4)$ & $0.5(0.4-0.6)$ & $0.3(0.3-0.4)$ & $0.2(0.1-0.3)$ & $0.2(0.1-0.3)$ \\
\hline
\end{tabular}

Table 2. Number of individuals $(n)$ and basal area $\left(A B-m^{2}\right)$ of tree species sampled in a 10.24-ha plot of tropical semi-deciduous forest in the Caetetus Ecological Station. Voucher cited as collector or collection number (ESA Herbarium). Collectors: F - G.A.D.C. Franco, G - Maurício Gorenstein, P- F.C. Passos, S - M. Silvestrini, T - M.T.Z. Toniato. * Alien species. FC - leaf fall category: E = evergreen, $\mathrm{D}=$ deciduous, $\mathrm{S}=$ semi-deciduous.

\begin{tabular}{|c|c|c|c|c|}
\hline Family / Species & Voucher & FC & $n$ & AB \\
\hline \multicolumn{5}{|l|}{ Anacardiaceae } \\
\hline Astronium graveolens Jacq. & F 4526 & $\mathrm{D}$ & 159 & 7.02 \\
\hline \multicolumn{5}{|l|}{ Annonaceae } \\
\hline Annona cacans Warm. & ESA105383 & $\mathrm{D}$ & 2 & 0.10 \\
\hline Annona emarginata (Schltdl.) H.Rainer & F 4635 & $\mathrm{D}$ & 1 & 0.02 \\
\hline Annona sylvatica A.St.-Hil. & F 4613 & $\mathrm{E}$ & 14 & 0.19 \\
\hline \multicolumn{5}{|l|}{ Apocynaceae } \\
\hline Aspidosperma polyneuron Müll.Arg. & T 58 & $\mathrm{E}$ & 643 & 40.78 \\
\hline Tabernaemontana catharinensis A. DC. & F 4540 & $\mathrm{E}$ & 7 & 0.02 \\
\hline \multicolumn{5}{|l|}{ Araliaceae } \\
\hline Aralia warmingiana (Marchal) J.Wen & T 70 & $\mathrm{D}$ & 3 & 0.08 \\
\hline \multicolumn{5}{|l|}{ Arecaceae } \\
\hline Euterpe edulis Mart. & & $\mathrm{E}$ & 17 & 0.15 \\
\hline Syagrus oleracea (Mart.) Becc. & G 5034 & $\mathrm{E}$ & 133 & 9.61 \\
\hline Syagrus romanzoffiana (Cham.) Glassman & G 5002 & $\mathrm{E}$ & 286 & 0.13 \\
\hline \multicolumn{5}{|l|}{ Asteraceae } \\
\hline Piptocarpha sellowii (Sch.Bip.) Baker & F 4646 & S & 6 & 0.05 \\
\hline Vernonanthura divaricata (Spreng.) H. Rob. & F 4585 & $\mathrm{D}$ & 2 & 0.00 \\
\hline Vernonanthura polyanthes (Spreng.) Less. & P 33 & $\mathrm{D}$ & 1 & 0.00 \\
\hline \multicolumn{5}{|l|}{ Bignoniaceae } \\
\hline Handroanthus umbellatus (Sond.) Mattos & F 4529 & $\mathrm{D}$ & 1 & 0.00 \\
\hline Jacaranda micrantha Cham. & F 4508 & $\mathrm{D}$ & 21 & 0.58 \\
\hline Zeyheria tuberculosa (Vell.) Bureau ex Verl. & F 4562 & $\mathrm{D}$ & 13 & 0.39 \\
\hline \multicolumn{5}{|l|}{ Boraginaceae } \\
\hline Cordia americana (L.) Gottschling \& J.S.Mill. & F 4567 & $\mathrm{D}$ & 26 & 5.04 \\
\hline Cordia ecalyculata Vell. & F 4624 & $\mathrm{E}$ & 59 & 1.02 \\
\hline Cordia superba Cham. & F 4505 & $S$ & 13 & 0.45 \\
\hline Cordia trichotoma (Vell.) Arráb. ex Steud. & F 4549 & $\mathrm{D}$ & 5 & 0.07 \\
\hline \multicolumn{5}{|l|}{ Cannabaceae } \\
\hline Trema micrantha (L.) Blume & ESA105389 & $\mathrm{E}$ & 5 & 0.01 \\
\hline
\end{tabular}


Tiago Egydio Barreto, Natália Macedo Ivanauskas, Renato Augusto Ferreira de Lima, Maria Teresa Zugliani Toniato, Flaviana Maluf Souza and Ricardo Ribeiro Rodrigues

Table 2. Cont.

\begin{tabular}{|c|c|c|c|c|}
\hline Family / Species & Voucher & FC & $n$ & AB \\
\hline \multicolumn{5}{|l|}{ Cardiopteridaceae } \\
\hline Citronella paniculata (Mart.) R.A.Howard & Т 79 & $S$ & 16 & 0.10 \\
\hline \multicolumn{5}{|l|}{ Caricaceae } \\
\hline Jacaratia spinosa (Aubl.) A.DC. & F 4555 & $\mathrm{D}$ & 27 & 2.07 \\
\hline \multicolumn{5}{|l|}{ Celastraceae } \\
\hline Monteverdia aquifolia (Mart.) Biral & F 4546 & $\mathrm{E}$ & 2 & 0.02 \\
\hline Monteverdia gonoclada (Mart.) Biral & F 4519 & $S$ & 4 & 0.03 \\
\hline \multicolumn{5}{|l|}{ Ebenaceae } \\
\hline Diospyros inconstans Jacq. & F 4510 & $\mathrm{E}$ & 1 & 0.00 \\
\hline \multicolumn{5}{|l|}{ Elaeocarpaceae } \\
\hline Sloanea lasiocoma K.Schum. & F 4544 & $\mathrm{E}$ & 4 & 0.04 \\
\hline \multicolumn{5}{|l|}{ Euphorbiaceae } \\
\hline Actinostemon concepcionis (Chodat \& Hassl.)Hochr. & F 4609 & $\mathrm{D}$ & 22 & 0.10 \\
\hline Actinostemon concolor (Spreng.) Müll.Arg. & F 4553 & $\mathrm{D}$ & 68 & 0.26 \\
\hline Alchornea glandulosa Poepp. \& Endl. & T 73 & $\mathrm{E}$ & 6 & 0.48 \\
\hline Croton floribundus Spreng. & S 3 & S & 510 & 12.17 \\
\hline Pachystroma longifolium (Nees) I.M.Johnst. & G 8180 & $\mathrm{E}$ & 1 & 0.10 \\
\hline Sapium glandulosum (L.) Morong & F 4595 & $\mathrm{E}$ & 2 & 0.01 \\
\hline \multicolumn{5}{|l|}{ Fabaceae } \\
\hline Albizia niopoides (Spruce ex Benth.) Burkart & F 4580 & $\mathrm{D}$ & 3 & 0.13 \\
\hline Albizia polycephala (Benth.) Killip ex Record & F 4569 & $\mathrm{D}$ & 15 & 0.67 \\
\hline Bauhinia longifolia (Bong.) Steud. & F 4638 & $\mathrm{E}$ & 8 & 0.14 \\
\hline Calliandra foliolosa Benth. & F 4530 & $\mathrm{D}$ & 8 & 0.04 \\
\hline Cassia ferruginea (Schrad.) Schrad. ex DC. & G 14878 & $\mathrm{D}$ & 1 & 0.00 \\
\hline Centrolobium tomentosum Guillem. ex Benth. & F 4639 & $\mathrm{D}$ & 400 & 13.68 \\
\hline Enterolobium contortisiliquum (Vell.) Morong & G 1532 & $\mathrm{D}$ & 14 & 1.40 \\
\hline Holocalyx balansae Micheli & F 4515 & S & 104 & 3.19 \\
\hline Hymenaea courbaril L. & F 4511 & $\mathrm{D}$ & 2 & 0.02 \\
\hline Inga marginata Willd. & F 4535 & $\mathrm{E}$ & 19 & 0.07 \\
\hline Inga striata Benth. & F 4550 & $\mathrm{E}$ & 43 & 1.31 \\
\hline Lonchocarpus cultratus (Vell.) A.M.G.Azevedo \& H.C.Lima & F 4516 & $\mathrm{D}$ & 49 & 2.01 \\
\hline Machaerium hirtum (Vell.) Stellfeld & G 13166 & $\mathrm{D}$ & 5 & 0.09 \\
\hline Machaerium nyctitans (Vell.) Benth. & T 64 & $\mathrm{D}$ & 17 & 0.61 \\
\hline Machaerium stipitatum Vogel & F 4509 & S & 101 & 3.43 \\
\hline Muellera campestris (Mart. ex Benth.) M.J.Silva \& A.M.G.Azevedo & F 4527 & $\mathrm{D}$ & 1 & 0.08 \\
\hline Myroxylon peruiferum L.f. & F 4563 & $\mathrm{D}$ & 8 & 1.89 \\
\hline Ormosia arborea (Vell.) Harms & G 21524 & S & 1 & 0.16 \\
\hline Parapiptadenia rigida (Benth.) Brenan & T 80 & $\mathrm{D}$ & 40 & 4.47 \\
\hline Peltophorum dubium (Spreng.) Taub. & F 4568 & $\mathrm{D}$ & 20 & 1.95 \\
\hline Piptadenia gonoacantha (Mart.) J.F.Macbr. & F 4552 & $\mathrm{D}$ & 78 & 6.38 \\
\hline Schizolobium parahyba (Vell.) Blake * & & $\mathrm{D}$ & 2 & 0.33 \\
\hline Senegalia polyphylla (DC.) Britton \& Rose & G 5096 & $\mathrm{D}$ & 69 & 3.51 \\
\hline Senna multijuga (Rich.) H.S.Irwin \& Barneby & G 22771 & $\mathrm{D}$ & 1 & 0.00 \\
\hline Sweetia fruticosa Spreng. & F 4584 & $\mathrm{D}$ & 1 & 3.23 \\
\hline \multicolumn{5}{|l|}{ Lamiaceae } \\
\hline Aegiphila integrifolia (Jacq.) Moldenke & F 4611 & $\mathrm{D}$ & 2 & 0.01 \\
\hline Vitex megapotamica (Spreng.) Moldenke & F 4559 & $\mathrm{D}$ & 3 & 0.14 \\
\hline \multicolumn{5}{|l|}{ Lauraceae } \\
\hline Endlicheria paniculata (Spreng.) J.F.Macbr. & T 76 & $\mathrm{E}$ & 9 & 0.07 \\
\hline Nectandra megapotamica (Spreng.) Mez & F 4614 & E & 28 & 0.90 \\
\hline Ocotea diospyrifolia (Meisn.) Mez & F 4630 & $\mathrm{E}$ & 2 & 0.50 \\
\hline Ocotea prolifera (Nees \& Mart.) Mez & F 4500 & $\mathrm{E}$ & 665 & 18.59 \\
\hline Ocotea silvestris Vattimo-Gil & F 4574 & $\mathrm{E}$ & 3 & 0.11 \\
\hline
\end{tabular}


Table 2. Cont.

\begin{tabular}{|c|c|c|c|c|}
\hline Family / Species & Voucher & FC & $n$ & AB \\
\hline Ocotea velutina (Nees) Rohwer & F 4504 & $\mathrm{E}$ & 9 & 0.38 \\
\hline \multicolumn{5}{|l|}{ Lecythidaceae } \\
\hline Cariniana estrellensis (Raddi) Kuntze & F 4543 & $S$ & 16 & 0.99 \\
\hline \multicolumn{5}{|l|}{ Malvaceae } \\
\hline Ceiba speciosa (A.St.-Hil.) Ravenna & F 4592 & $\mathrm{D}$ & 36 & 3.97 \\
\hline Heliocarpus popayanensis Kunth & F 4541 & $\mathrm{D}$ & 4 & 0.11 \\
\hline Luehea divaricata Mart. \& Zucc. & ESA105396 & $\mathrm{D}$ & 1 & 0.13 \\
\hline \multicolumn{5}{|l|}{ Meliaceae } \\
\hline Cabralea canjerana (Vell.) Mart. & ESA105415 & $\mathrm{D}$ & 20 & 1.25 \\
\hline Cedrela fissilis Vell. & T 65 & $\mathrm{D}$ & 47 & 1.99 \\
\hline Guarea guidonia (L.) Sleumer & G 15914 & $\mathrm{E}$ & 1 & 0.00 \\
\hline Guarea kunthiana A.Juss. & F 4587 & $\mathrm{E}$ & 6 & 0.06 \\
\hline Guarea macrophylla Vahl & & $\mathrm{E}$ & 1 & 0.00 \\
\hline Trichilia casaretti C.DC. & F 4577 & $\mathrm{E}$ & 2 & 2.97 \\
\hline Trichilia catigua A.Juss. & T 71 & $\mathrm{E}$ & 590 & 4.39 \\
\hline Trichilia clausseni C.DC. & F 4521 & $\mathrm{E}$ & 602 & 0.49 \\
\hline Trichilia pallida Sw. & F 4621 & $\mathrm{E}$ & 57 & 0.74 \\
\hline \multicolumn{5}{|l|}{ Monimiaceae } \\
\hline Mollinedia widgrenii A.DC. & F 4524 & S & 37 & 0.58 \\
\hline \multicolumn{5}{|l|}{ Moraceae } \\
\hline Ficus eximia Schott & F 4632 & $\mathrm{D}$ & 5 & 0.07 \\
\hline Ficus lagoensis C.C.Berg \& Carauta & T 69 & $\mathrm{D}$ & 4 & 4.33 \\
\hline Ficus luschnathiana (Miq.) Miq. & SPSF 13506 & $S$ & 6 & 3.53 \\
\hline Ficus sp. & & S & 5 & 0.49 \\
\hline Maclura tinctoria (L.) D.Don ex Steud. & F 4539 & $\mathrm{D}$ & 1 & 0.07 \\
\hline Sorocea bonplandii (Baill.) W.C.Burger et al. & F 4629 & $\mathrm{E}$ & 13 & 0.07 \\
\hline \multicolumn{5}{|l|}{ Myrtaceae } \\
\hline Campomanesia guazumifolia (Cambess.) O.Berg & T 67 & $\mathrm{D}$ & 6 & 0.05 \\
\hline Campomanesia xanthocarpa (Mart.) O.Berg & F 4631 & $\mathrm{D}$ & 100 & 1.88 \\
\hline Eugenia florida DC. & F 4603 & $\mathrm{E}$ & 8 & 0.05 \\
\hline Eugenia longipedunculata Nied. & F 4572 & $\mathrm{E}$ & 30 & 0.19 \\
\hline Eugenia ramboi D.Legrand & F 4512 & $\mathrm{E}$ & 178 & 1.52 \\
\hline Eugenia subterminalis DC. & Т 78 & $\mathrm{E}$ & 21 & 0.06 \\
\hline Eugenia uniflora L. & F 4514 & $\mathrm{E}$ & 1 & 0.05 \\
\hline Myrcianthes pungens (O.Berg) D.Legrand & F 4599 & S & 3 & 0.03 \\
\hline Myrciaria floribunda (H.West. ex Willd) O.Berg & T 81 & $S$ & 5 & 0.02 \\
\hline Neomitranthes glomerata (D.Legrand) D.Legrand & F 4601 & $\mathrm{E}$ & 54 & 0.48 \\
\hline Plinia peruviana (Poir.) Govaerts & F 4605 & S & 9 & 0.34 \\
\hline Plinia rivularis (Cambess.) Rotman & & $\mathrm{E}$ & 1 & 0.00 \\
\hline Psidium sartorianum (O.Berg) Nied. & F 4545 & $\mathrm{E}$ & 2 & 0.03 \\
\hline \multicolumn{5}{|l|}{ Nyctaginaceae } \\
\hline Bougainvillea glabra Choi sy & F 4571 & $\mathrm{E}$ & 9 & 0.67 \\
\hline Guapira hirsuta (Choisy) Lundell & T 82 & $\mathrm{E}$ & 6 & 0.03 \\
\hline Guapira opposita (Vell.) Reitz & G 17571 & $\mathrm{E}$ & 2 & 0.01 \\
\hline Pisonia ambigua Heimerl & F 4596 & $\mathrm{E}$ & 10 & 0.60 \\
\hline \multicolumn{5}{|l|}{ Opiliaceae } \\
\hline Agonandra excelsa Griseb. & F 4583 & $\mathrm{D}$ & 19 & 0.29 \\
\hline \multicolumn{5}{|l|}{ Phyllanthaceae } \\
\hline Margaritaria nobilis L.f. & $\mathrm{T} 61$ & $\mathrm{D}$ & 7 & 0.09 \\
\hline Savia dictyocarpa Müll.Arg. & F 4608 & S & 162 & 5.85 \\
\hline \multicolumn{5}{|l|}{ Phytolaccaceae } \\
\hline Gallesia integrifolia (Spreng.) Harms & F 4637 & $\mathrm{E}$ & 32 & 5.62 \\
\hline Seguieria aculeata Jacq. & F 4623 & $\mathrm{E}$ & 40 & 0.40 \\
\hline
\end{tabular}


Tiago Egydio Barreto, Natália Macedo Ivanauskas, Renato Augusto Ferreira de Lima, Maria Teresa Zugliani Toniato, Flaviana Maluf Souza and Ricardo Ribeiro Rodrigues

Table 2. Cont.

\begin{tabular}{|c|c|c|c|c|}
\hline Family / Species & Voucher & FC & n & AB \\
\hline \multicolumn{5}{|l|}{ Picramniaceae } \\
\hline Picramnia glazioviana Engl. & & S & 4 & 0.02 \\
\hline Picramnia ramiflora Planch. & F 4627 & $S$ & 22 & 0.10 \\
\hline \multicolumn{5}{|l|}{ Piperaceae } \\
\hline Piper amalago $\mathrm{L}$. & F 4534 & $\mathrm{E}$ & 8 & 0.03 \\
\hline \multicolumn{5}{|l|}{ Polygonaceae } \\
\hline Ruprechtia laxiflora Meisn. & F 4528 & $S$ & 1 & 0.06 \\
\hline \multicolumn{5}{|l|}{ Primulaceae } \\
\hline Myrsine lancifolia Mart. & F 4513 & $\mathrm{E}$ & 3 & 0.03 \\
\hline Myrsine umbellata Mart. & G 15262 & $\mathrm{E}$ & 7 & 0.37 \\
\hline \multicolumn{5}{|l|}{ Proteaceae } \\
\hline Roupala montana (Klotzsch) K.S.Edwards & F 4502 & $\mathrm{D}$ & 2 & 0.03 \\
\hline \multicolumn{5}{|l|}{ Rhamnaceae } \\
\hline Colubrina glandulosa Perkins & F 4576 & $\mathrm{D}$ & 11 & 0.47 \\
\hline Rhamnidium elaeocarpum Reissek & F 4591 & $\mathrm{D}$ & 46 & 0.91 \\
\hline \multicolumn{5}{|l|}{ Rubiaceae } \\
\hline Coutarea hexandra (Jacq.) K.Schum. & F 4554 & $\mathrm{E}$ & 2 & 0.05 \\
\hline Ixora venulosa Benth. & F 4640 & $\mathrm{E}$ & 4 & 0.02 \\
\hline Randia calycina Cham. & F 4579 & $\mathrm{D}$ & 1 & 0.00 \\
\hline Rudgea jasminoides (Cham.) Müll.Arg. & G 17249 & $\mathrm{E}$ & 1 & 0.01 \\
\hline \multicolumn{5}{|l|}{ Rutaceae } \\
\hline Balfourodendron riedelianum (Engl.) Engl. & T 75 & $\mathrm{D}$ & 327 & 8.23 \\
\hline Esenbeckia leiocarpa Engl. & F 4642 & $S$ & 187 & 4.34 \\
\hline Metrodorea nigra A.St.-Hil. & G 139 & $\mathrm{E}$ & 4394 & 30.96 \\
\hline Pilocarpus pauciflorus A.St.-Hil. & F 4578 & $\mathrm{E}$ & 45 & 0.29 \\
\hline Pilocarpus pennatifolius Lem. & F 4641 & $\mathrm{E}$ & 5 & 0.01 \\
\hline Zanthoxylum caribaeum Lam. & F 4628 & S & 13 & 1.08 \\
\hline Zanthoxylum fagara (L.) Sarg. & T 62 & $\mathrm{D}$ & 23 & 0.30 \\
\hline Zanthoxylum rhoifolium Lam. & F 4612 & $\mathrm{D}$ & 7 & 0.15 \\
\hline Zanthoxylum riedelianum Engl. & F 4560 & $\mathrm{D}$ & 8 & 0.06 \\
\hline \multicolumn{5}{|l|}{ Salicaceae } \\
\hline Casearia decandra Jacq. & G 23619 & $\mathrm{D}$ & 1 & 0.00 \\
\hline Casearia gossypiosperma Briq. & T 63 & $\mathrm{D}$ & 68 & 0.66 \\
\hline Casearia sylvestris $\mathrm{Sw}$. & F 4589 & $\mathrm{E}$ & 44 & 0.42 \\
\hline Prockia crucis P.Browne ex L. & Т 77 & S & 5 & 0.05 \\
\hline Xylosma tweediana (Clos) Eichler & F 4593 & S & 1 & 0.01 \\
\hline \multicolumn{5}{|l|}{ Sapindaceae } \\
\hline Allophylus edulis (A.St.-Hil. et al.) Hieron. ex Niederl. & Т 59 & S & 3 & 0.04 \\
\hline Cupania vernalis Cambess. & F 4598 & S & 41 & 0.57 \\
\hline Diatenopteryx sorbifolia Radlk. & F 4625 & S & 47 & 1.48 \\
\hline Matayba elaeagnoides Radlk. & T 74 & S & 1 & 0.00 \\
\hline \multicolumn{5}{|l|}{ Sapotaceae } \\
\hline Chrysophyllum gonocarpum (Mart. \& Eichler ex Miq.) Engl. & F 4588 & $\mathrm{E}$ & 218 & 7.00 \\
\hline \multicolumn{5}{|l|}{ Solanaceae } \\
\hline Cestrum strigilatum Ruiz \& Pav. & F 4634 & $\mathrm{E}$ & 1 & 0.00 \\
\hline Solanum argenteum Duna & T 60 & S & 22 & 0.19 \\
\hline Solanum pseudoquina A.St.-Hil. & F 4547 & S & 1 & 0.01 \\
\hline \multicolumn{5}{|l|}{ Urticaceae } \\
\hline Cecropia glaziovii Snethl. & G 5984 & $\mathrm{E}$ & 14 & 0.49 \\
\hline Urera baccifera (L.) Gaudich. ex Wedd. & F 4633 & $\mathrm{D}$ & 26 & 0.11 \\
\hline \multicolumn{5}{|l|}{ Verbenaceae } \\
\hline Aloysia virgata (Ruiz \& Pav.) Juss. & F 4606 & $\mathrm{D}$ & 1 & 0.00 \\
\hline
\end{tabular}




\section{Canopy openness and soil conditions explain community structure and diversity in a tropical seasonal forest in south-eastern Brazil}

Table 3. The 10 high-density species (ind. ha-1) found in a 10.24 ha-plot of tropical semi-deciduous forest in the Caetetus Ecological Station. The species rank was ordered by the minor size class considered. Values outside the brackets correspond to the median while values in the brackets correspond to the first and third quartiles of the distribution.

\begin{tabular}{|c|c|c|c|c|c|}
\hline \multirow{2}{*}{ Species } & \multicolumn{5}{|c|}{ Size class $(\mathrm{cm})$} \\
\hline & $\mathrm{DBH} \geq 4.8$ & $4.8 \leq \mathrm{DBH}<10$ & $10 \leq \mathrm{DBH}<20$ & $20 \leq \mathrm{DBH}<30$ & $\mathrm{DBH} \geq 30$ \\
\hline Metrodorea nigra & $455(415-473)$ & $339(284-352)$ & $113(107-117)$ & $6(3-8)$ & \\
\hline Ocotea prolifera & $69(59-78)$ & $20(16-32)$ & $26(23-31)$ & $9(7-14)$ & $5(2-11)$ \\
\hline Trichilia clausseni & $67(52-73)$ & $46(33-52)$ & $19(14-21)$ & & \\
\hline Aspidosperma polyneuron & $62(54-76)$ & $25(22-34)$ & $21(16-24)$ & $5(3-5)$ & $12(10-15)$ \\
\hline Trichilia catigua & $63(48-71)$ & $53(40-60)$ & & & \\
\hline Centrolobium tomentosum & $39(33-50)$ & $11(8-17)$ & $12(8-18)$ & $9(7-11)$ & $6(4-7)$ \\
\hline Croton floribundus & $54(36-61)$ & $22(16-24)$ & $20(15-24)$ & $8(5-10)$ & $4(3-6)$ \\
\hline Balfourodendron riedelianum & $31(20-42)$ & $16(8-24)$ & $9(7-12)$ & $1(1-3)$ & $3(2-5)$ \\
\hline Syagrus romanzoffiana & $29(22-37)$ & & $12(7-14)$ & $17(14-22)$ & \\
\hline Chrysophyllum gonocarpum & $22(19-26)$ & & $12(10-14)$ & $7(5-9)$ & \\
\hline Eugenia ramboi & & $12(8-16)$ & & & \\
\hline Syagrus oleracea & & & $10(8-16)$ & & \\
\hline Astronium graveolens & & & & $5(3-8)$ & $2(1-3)$ \\
\hline Esenbeckia leiocarpa & & $0(0-3)$ & & $0(0-2)$ & \\
\hline Savia dictyocarpa & & & & & $3(2-4)$ \\
\hline Gallesia integrifolia & & & & & $2(1-3)$ \\
\hline Senegalia polyphylla & & & & & $1(0-4)$ \\
\hline Piptadenia gonoacantha & & & & & $1(0-2)$ \\
\hline
\end{tabular}

of individuals sampled, with a marked influence of species typical from the understory (Tab. 3). Many of the common species in the canopy (mentioned above) also presented the largest diameters. However, less common species in the upper strata had large contributions in the plot basal area, such as Astronium graveolens, Savia dictyocarpa, Gallesia integrifolia, Senegalia polyphylla and Piptadenia gonoacantha.

\section{The influence of canopy openness and soil conditions}

The DBH cutoff criterion was critical to the evaluation of the environmental heterogeneity effect on the community descriptors at the 8.96-ha scale (Tab. 4). The null model was the best fit to the community data for most part of descriptors studied for DBH $\geq 4.8 \mathrm{~cm}$, with exception of basal area and the density of deciduous species, for which the model including canopy openness (light regime model) was the best fit (Tab. 4). Canopy openness was significant and inversely proportional with basal area, and light regime and base saturation were significant and directly proportional with the density of deciduous trees (Tab. 5). The canopy openness and soil properties effects were more pronounced when we considered only individuals with $\mathrm{DBH} \geq 20 \mathrm{~cm}$, which encompasses the upper forest strata (Tab. 4). Light regime was significantly and inversely proportional to the forest structure descriptors (basal area, density) and diversity (richness and Simpson diversity). Regarding the edaphic parameters, phosphorus was significantly and positively correlated to basal area and tree density. Organic matter was significantly and negatively correlated to tree density. Finally, base saturation was significantly and negatively correlated to the density of semi-deciduous trees (Tab. 5).

\section{Discussion}

\section{General description of forest structure and diversity}

Tree density and basal area found in the studied forest (1,129 ind. ha ${ }^{-1}$ and $24.8 \mathrm{~m}^{2} \mathrm{ha}^{-1}-$ Tab. 1$)$ were lower than in other seasonal forests from southeastern Brazil, ranging from 1,280 to 3,637 ind. ha ${ }^{-1}$ and 28.7 to $40.2 \mathrm{~m}^{2} \mathrm{ha}^{-1}$ (Oliveira-Filho \& Machado 1993; Oliveira-Filho et al. 1994; Ivanauskas et al. 1999; Fonseca \& Rodrigues 2000; Durigan et al. 2000; Botrel et al. 2002; Silva et al. 2003; Silva et al. 2004; Santos et al. 2012a). Our tree density estimates were only higher than one conducted in another part of the same conservation unit (1,080 ind. ha ${ }^{-1}-$ Durigan et al. 2000). Although tree density may vary along forest succession, old growth forests have higher basal area, suggesting that our 10-ha plot may have suffered from disturbances. The permanent plot studied have no recent evidence of humanrelated disturbances (Botrel et al. 2013). However, Lima et al. (2008) argued that gap density and area in the plot was markedly higher than other tropical forests, suggesting that a catastrophic disturbance event probably occurred. The only known disturbance in the area is a wind corridor responsible for the frequent fall of tall trees, a common phenomenon in the area (Barreto 2015).

We found only one non-native species in the plot, Schizolobium parahyba, which does not occur naturally in the conservation unit but is cultivated nearby (Durigan et al. 2013). The number of rare species (i.e., singletons) per hectare represented $19 \%$ of the plot richness, which is smaller than in the observed in other semi-deciduous 
Tiago Egydio Barreto, Natália Macedo Ivanauskas, Renato Augusto Ferreira de Lima, Maria Teresa Zugliani Toniato, Flaviana Maluf Souza and Ricardo Ribeiro Rodrigues

Table 4. Results from the multiple linear regression analysis of the structure and diversity of a tropical semi-deciduous forest in the Caetetus Ecological Station. $\triangle \mathrm{AIC}$ values (the larger number from which we subtracted the smaller Akaike information criterion - AIC) greater than or equal to eight indicates differences between models that are statistically significant. The model with the lowest $\triangle \mathrm{AIC}$ value is considered the model with best performance (highlighted in bold). DBH = Diameter at Breast Height.

\begin{tabular}{|c|c|c|c|c|c|c|c|}
\multirow{2}{*}{ Variables responses } & \multicolumn{3}{|c|}{ DBH $\geq 4.8 \mathrm{~cm}$} & \multicolumn{4}{c|}{ DBH $\geq 20 \mathrm{~cm}$} \\
\cline { 2 - 7 } & \multicolumn{3}{|c|}{$\Delta$ AIC } & Light & Null Model & Soil & Light \\
\cline { 2 - 7 } & Null Model & Soil & 6.6 & $\mathbf{0 . 0}$ & 8.1 & 6.3 & $\mathbf{0 . 0}$ \\
\hline Basal area & 7.0 & 4.2 & 4.1 & 20.3 & 20.2 & $\mathbf{0 . 0}$ \\
\hline Density & $\mathbf{0 . 0}$ & 4.2 & 3.0 & $\mathbf{0 . 0}$ & 7.2 & 8.3 \\
\hline Evergreen density & $\mathbf{0 . 0}$ & 2.3 & $\mathbf{0 . 0}$ & $\mathbf{0 . 0}$ & 2.4 & 4.4 \\
\hline Deciduous density & 2.5 & 1.5 & 3.4 & 2.6 & $\mathbf{0 . 0}$ & 1.4 \\
\hline Semi-deciduous density & $\mathbf{0 . 0}$ & 3.1 & 4.0 & $\mathbf{0 . 0}$ & 4.2 & 5.8 \\
\hline Fisher's Alpha & $\mathbf{0 . 0}$ & 4.1 & 5.3 & 5.7 & 9.1 & $\mathbf{0 . 0}$ \\
\hline Simpson diversity & $\mathbf{0 . 0}$ & 2.7 & 4.4 & 10.9 & 13.6 & $\mathbf{0 . 0}$ \\
\hline Richness & $\mathbf{0 . 0}$ & & & & & \\
\hline
\end{tabular}

Table 5. T-test applied to the coefficient estimated in the optimum regression model, with the explanatory variables used in the regression analysis of the structure and diversity in a tropical semi-deciduous forest in the Caetetus Ecological Station. Only regression models with $\triangle \mathrm{AIC}$ values greater than $\log (8)$ are presented. Values outside the parentheses correspond to the $p$-values while values in the parentheses correspond to the $t$-test values. The significant regression parameters are highlighted in bold. DBH $=\mathrm{Diameter}$ at Breast Height; Index $\mathrm{S}=$ soil structure and porosity variable; $\mathrm{MO}=$ organic matter; $\mathrm{V} \%$ = base saturation; $\mathrm{P}=$ soil phosphorus content.

\begin{tabular}{|c|c|c|c|c|c|c|c|c|c|c|}
\hline \multirow{2}{*}{ Descriptors } & \multicolumn{5}{|c|}{$\mathrm{DBH} \geq 4.8 \mathrm{~cm}$} & \multicolumn{5}{|c|}{$\mathrm{DBH} \geq 20 \mathrm{~cm}$} \\
\hline & SIndex & MO & V \% & $P$ & Light & S Index & MO & $\mathbf{V} \%$ & $\mathbf{P}$ & Light \\
\hline Basal area & $\begin{array}{c}0.123 \\
(-1.54)\end{array}$ & $\begin{array}{l}0.808 \\
(0.24)\end{array}$ & $\begin{array}{l}0.668 \\
(0.43)\end{array}$ & $\begin{array}{l}0.093 \\
(1.69)\end{array}$ & $\begin{array}{c}0.004 \\
(-2.92)\end{array}$ & $\begin{array}{c}0.118 \\
(-1.57)\end{array}$ & $\begin{array}{c}0.978 \\
(-0.02)\end{array}$ & $\begin{array}{l}0.436 \\
(0.78)\end{array}$ & $\begin{array}{l}0.050 \\
(1.96)\end{array}$ & $\begin{array}{c}0.04 \\
(-2.86)\end{array}$ \\
\hline Density & & & & & & $\begin{array}{c}0.306 \\
(-1.02)\end{array}$ & $\begin{array}{c}0.047 \\
(-1.99)\end{array}$ & $\begin{array}{c}0.854 \\
(-0.18)\end{array}$ & $\begin{array}{l}0.033 \\
(2.14)\end{array}$ & $\begin{array}{l}<0.001 \\
(-4.70)\end{array}$ \\
\hline $\begin{array}{l}\text { Deciduous } \\
\text { density }\end{array}$ & $\begin{array}{l}0.743 \\
(0.32)\end{array}$ & $\begin{array}{c}0.118 \\
(-1.57)\end{array}$ & $\begin{array}{l}0.049 \\
(1.98)\end{array}$ & $\begin{array}{c}0.055 \\
(-1.93)\end{array}$ & $\begin{array}{l}0.039 \\
(2.07)\end{array}$ & & & & & \\
\hline $\begin{array}{c}\text { Semi-deciduous } \\
\text { density }\end{array}$ & & & & & & $\begin{array}{c}0.253 \\
(-1.14)\end{array}$ & $\begin{array}{l}0.068 \\
(1.83)\end{array}$ & $\begin{array}{c}0.014 \\
(-2.48)\end{array}$ & $\begin{array}{l}0.212 \\
(1.25)\end{array}$ & $\begin{array}{c}0.235 \\
(-1.19)\end{array}$ \\
\hline $\begin{array}{l}\text { Simpson } \\
\text { diversity }\end{array}$ & & & & & & $\begin{array}{c}0.115 \\
(-1.58)\end{array}$ & $\begin{array}{c}0.293 \\
(-1.05)\end{array}$ & $\begin{array}{c}0.805 \\
(-0.25)\end{array}$ & $\begin{array}{l}0.501 \\
(0.67)\end{array}$ & $\begin{array}{r}0.001 \\
(-3.41)\end{array}$ \\
\hline Richness & & & & & & $\begin{array}{c}0.143 \\
(-1.46)\end{array}$ & $\begin{array}{c}0.292 \\
(-1.05)\end{array}$ & $\begin{array}{l}0.862 \\
(0.17)\end{array}$ & $\begin{array}{l}0.388 \\
(0.86)\end{array}$ & $\begin{array}{l}0.0001 \\
(-3.97)\end{array}$ \\
\hline
\end{tabular}

seasonal forests (> 26\%, Santos et al. 2013). The Caetetus 10-ha plot was characterized by a relatively low diversity (H' 2.84) and equability (0.64) per hectare, which is related to the high abundance of one understory species, Metrodorea nigra. This result is consistent with the one found in another part of the same forest fragment (Durigan et al. 2000).

In a forest in dynamic equilibrium, the community structure should be characterized by a high number of small plants in the understory and a decreasingly smaller number of trees towards the upper strata of the forest (Pires \& Prance 1977). However, the abundance of M. nigra in Caetetus understory was markedly elevated and had direct consequences in the local diversity. The ecological dominance of few understory species it is not uncommon in seasonal forests, although the species identity may shift between localities (Botrel et al. 2002). Considering out plot as part of the seasonally dry tropical forests (SDTF) sensu lato (Linares-Palomino et al. 2011; Leigh-Jr 2019), there is little evidence for any oligarchy of species that dominates across SDTFs, with higher species turnover at continental, regional, and local scales (DRYFLOR 2016). In addition,
SDTF specialists tend to be locally abundant, since they are adapted to the limited opportunities for successful establishment (DRYFLOR 2016).

A high abundance of $M$. nigra has already been recorded in other seasonal forests of São Paulo state (Metzger et al. 1998; Silva \& Soares 2002). It is a small, evergreen tree frequently found in moist and semi-deciduous forests in eastern South America, from south to northeast Brazil (Pirani \& Skorupa 2002; Souza et al. 2004). The species is described as a self-incompatible allogamous, pollinated by flies, autochoric, dispersing its seeds via explosive dehiscence (Pombal \& Morellato 2000; Schwarcz et al. 2010). Its presence can indicate good levels of conservation, because it grows preferably in the shade and innermost preserved regions of the forests (Alzate-Marin et al. 2016), and habitat reduction has little effect on the genetic variability of $M$. nigra, since that larger fragments do not necessarily contain populations with greater genetic diversity (Moraes-Filho et al. 2015). Its high abundance in our site suggests that this species can cope with both seasonal climate of semi-deciduous forests, maybe presenting an ability to re-sprout after damage (personal 


\section{Canopy openness and soil conditions explain community structure and diversity in a tropical seasonal forest in south-eastern Brazil}

observations), which would be advantageous for understory species (Paciorek et al. 2000; Martini et al. 2008).

\section{The influence of canopy openness and soil conditions}

Overall, we found that small-scale variation of canopy openness and soil conditions did affect the structure and diversity of the tree community both size classes evaluated (i.e., $\mathrm{DBH} \geq 4.8$ and $\geq 20 \mathrm{~cm}$, Tab. 5). The midstory and understory layers ( $\mathrm{DBH}<20 \mathrm{~cm}$ ) were dominated notably by Metrodorea nigra, Trichilia clausseni and Trichilia catigua, with $M$. nigra representing almost $40 \%$ of all individuals in the plot. These three species together account for almost $60 \%$ of all individuals sampled. The density is crucial: if it is too low or too high, we could not expect them to produce the correct model. The results were more pronounced when we considered only individuals with $\mathrm{DBH} \geq 20 \mathrm{~cm}$, which encompasses the upper forest strata.

Tree density, basal area, richness, and diversity were smaller in areas with more canopy gaps and thus higher light incidence. Lower density and basal area are expected for $\mathrm{DBH} \geq 20 \mathrm{~cm}$, because canopy openings are intrinsically related to the death of one or more canopy individuals (Lima et al. 2008; Sande et al. 2016). However, canopy gaps are expected to enhance the density of understory vegetation (Dupuy \& Chazdon 2008), an expectation which was not confirmed this expectation. On the other hand, the literature suggests that canopy gaps can increase alpha diversity (see Wright 2002 and references therein), a result which was also not observed here. These somehow contradictory results may suggest that gaps may play a more important role in changing species composition (Brokaw 1985; 1987; Denslow 1987; Peters 2003; Swamy \& Terborgh 2010; Obianga et al. 2014) and thus in the maintenance of beta than alpha diversity. Anyway, this result supports the importance of the canopy gaps to the structure and diversity of tropical forests (Wright 2002; Zimmerman \& Kormos 2012; Botrel et al. 2013).

The base saturation and light regime were related to increases in the density of deciduous trees. The strong connection between base saturation and deciduous trees is well known in the literature (Oliveira-Filho et al. 2006; Santos et al. 2012b; Rossatto et al. 2015; Turner et al. 2018) and "gaps of deciduousness" (sensu Gandolfi et al. 2007) creates different light regimes beneath deciduous trees (Gandolfi et al. 2009). Since tree species in seasonal forests respond differently to distinct levels of light (Souza \& Válio 2001; 2003), deciduous canopy trees may alter the germination, growth, stress, and death of species attempting to regenerate underneath them (Gandolfi et al. 2009). According to Givnish (2002), deciduous species should gain an edge over evergreen species because the last take several years to accumulate a full set of leaf cohorts. Deciduous species grow faster and have shorter leaf lifespan associated with high specific leaf areas values, than results obtained in lower leaf mass fraction (Kunstler et al. 2016). Early leafing provides saplings of deciduous trees an extra few days or weeks of photosynthesis under the deciduous canopy during the dry season, and that extra "carbon subsidy" can enable them to endure microsites that are shadier during the wet season (Givnish 1988; King 1994).

Organic matter is an important source of phosphorous in tropical forests, and there are strong correlations between biomass accumulation and phosphorus availability in the soil or leaves (Aragão et al. 2009; Quesada et al. 2009; Quesada et al. 2012). In fact, phosphorus availability increased forest density and biomass in Caetetus. Phosphorus is essential to plant growth but is commonly not available to roots given the high rates of absorption and its low mobility (Porder et al. 2007; Cernusak et al. 2010 Vitousek et al. 2010). Thus, phosphorous can contribute more to the growth of already established individuals or to species more efficient in phosphorus recruiting (Gleason et al. 2009; Turner et al. 2018). Tang et al. (2018) suggest that there are different adaptive abilities of deciduous and perennial plants to reallocate phosphorus and nitrogen in plant tissues, and perennial plants in some cases may be more successful in producing biomass.

Contrary to our expectations, soil structure and its potential to store water, described here by the S-index (Dexter 2004), did not had an effect on the density of deciduous trees. We expected an increase in the density of deciduous species in areas with lower water storage (AguirreGutierrez et al. 2019). Our results were similar to those found by Sande et al. (2016) that reported an increase in wood density, but not in the percentage of deciduous trees in drier sites of old-growth Neotropical forests.

According to Givnish (2002), greater soil infertility, rooting depth and permeability can favor the evergreen habit. Besides that, the author suggests that deciduous trees may demand more phosphorus given their more frequent leaf replacement. The competition for soil phosphorous by $\mathrm{N}$-fixing tropical Leguminosae should lead to shallow rooting and to a high incidence of deciduousness, especially when such rooting is combined with a well illuminated position high in the canopy, which in turn should be favored by the high leaf nitrogen levels associated with $\mathrm{N}_{2}$-fixation (Givnish 1999; Vargas et al. 2015). We did not find a strong correlation between deciduous trees and phosphorus in the Caetetus 10-ha plot, despite the fact that legumes represented $65 \%$ of the deciduous trees in the plot (Tab.2), even though not all them fix nitrogen. A study of Neotropical forests reported no increase in nitrogen fixers as Fabaceae in drier sites (Sande et al. 2016).

Phosphorus contributes to both rooting and a better use of water (Lopes et al. 1998; Cernusak et al. 2010), which can increase canopy tree resistance to prolonged drought episodes. Well-drained soils (Arenic Haplustult and Arenic Haplustalf) are predominant in the studied plot and are characterized by an abruptic textural gradient (i.e., clayrich $B$ Horizon), which favors water retention above the 


\section{Tiago Egydio Barreto, Natália Macedo Ivanauskas, Renato Augusto Ferreira de Lima, Maria Teresa Zugliani Toniato, Flaviana Maluf Souza and Ricardo Ribeiro Rodrigues}

point of permanent plant wilting, even during prolonged drought episodes (Cooper et al. 2012).

Therefore, soil water retention may not be variable enough at the scale of our plot to influence forest structure and diversity. However, the composition and structure of tropical forests may be altered by extreme drought events and general rises in temperature. Recent research has demonstrated that drought resistance is often related to tree size, with taller trees being more vulnerable to droughtinduced mortality (Esquivel-Muelbert et al. 2017), leading to the death of species that are predominantly found in wetter climates. Predicting the vulnerability to droughts and responses to future climate change in the tropics is one of the aims of a permanent plot. On a long term, we hope that this plot will contribute to the better understanding of the community dynamics and to assess if drought-intolerant species are more prone to be locally extinct in the seasonal Atlantic Forest remnants.

\section{Conclusion}

In this study we combined data from one of the largest plot inventories ever carried in tropical semi-deciduous forests (10.24 ha) with detailed data from canopy openness and soil physical-chemical properties. We showed that the structure (tree density and basal area) and the composition of the forest (density of deciduous species) were related to small scale variations in both canopy structure and soil properties, while species diversity was related only to the canopy structure. Therefore, we show variations in canopy structure and soil conditions are associated with smallscale changes in the community. Contrary to our initial expectation, the density of deciduous species was related to soil nutrients and not to soil water retention capacity.

\section{Acknowledgements}

We thank Fundação Florestal for the access to the study area and who contributed to plot data collection and organization. We are grateful to the specialists who helped identify species, namely Fiorella F. Mazine, Geraldo A.D.C. Franco, Giselda Durigan, João B. Baitello, Osny T. Aguiar. This work was supported by grants 1999/09635-0 and 2013/50718-5, São Paulo Research Foundation (FAPESP), and 561897/2010-7, Brazil, National Council for Scientific and Technological Development (CNPq).

\section{References}

Aguirre-Gutiérrez J, Oliveras I, Rifai S, et al. 2019. Drier tropical forests are susceptible to functional changes in response to a long-term drought. Ecology Letters 22: 855-865.

Alzate-Marin AL, Bonifacio-Anacleto F, de Moraes-Filho RM, Machado GP, Nazareno AG. 2016. Genetic analysis across the life stages of Metrodorea nigra (Rutaceae) in a population located in one urban landscape of Southeastern Brazil using a new set of microsatellite markers. Brazilian Journal of Botany 39: 795-799.

Aragão LEOC, Malhi Y, Metcalfe DB, et al. 2009. Above-and below-ground net primary productivity across ten Amazonian forests on contrasting soils. Biogeosciences 6: 2759-2778.

Barreto TE. 2015. Padrões e processos que influenciam a dinâmica e a estrutura das florestas estacionais semideciduais, SE, Brasil. 2015. $\mathrm{PhD}$ Thesis, Universidade de Campinas, Campinas.

Bolker B. 2009. bbmle: Tools for general maximum likelihood estimation. R package version 0.9.3. http://CRAN.R-project.org/package=bbmle. 25 Jan. 2014.

Botrel RT, Oliveira-Filho AT, Rodrigues LA, Curi N. 2002. Influência do solo e topografia sobre as variações da composição florística e estrutura da comunidade arbóreo-arbustiva de uma floresta estacional semidecidual em Ingaí, MG. Revista Brasileira de Botânica 25: 195-213.

Botrel RT, Yamamoto K, Rodrigues RR. 2013. Avaliação de um método de análise silvigênica em uma floresta estacional semidecidual. Ciência Florestal 23: 391-402.

Brenes-Arguedas T, Roddy AB, Coley PD, Kursar TA. 2011. Do differences in understory light contribute to species distributions along a tropical rainfall gradient? Oecologia 166: 443-456.

Brokaw NVL. 1985. Treefalls, regrowth and community structure in tropical forest. In: Picket STA, White PS. (eds.) The ecology of natural disturbance and patch dynamics. New York, Academic Press. p. 53-85.

Brokaw NVL. 1987. Gap-phase regeneration of three pioneer tree species in a tropical forest. Journal of Ecology 75: 9-19.

Burnham KP, Anderson DR. 2002. Model selection and multimode inference: A practical Information- Theoretic Approach. 2nd. edn. Colorado, Springer.

Cernusak LA, Winter K, Turner BL. 2010. Leaf nitrogen to phosphorus ratios of tropical trees: experimental assessment of physiological and environmental controls. New Phytologist 185: 770-779.

Colwell RK, Coddington JA.1994. Estimating terrestrial biodiversity through extrapolation. Philosophical Transactions: Biological Sciences 345: 101-118.

Colwell RK. 2006. EstimateS: statistical estimation of species richness and shared species from samples. version 9. http://purl.oclc.org/ estimates. 25 Jan. 2014.

Condit R. 1998. Tropical forest census plots. Berlin, Springer-Verlag.

Cooper M, Dalla Rosa J, Medeiros JC, Oliveira T, Toma RS, Juhász CEP. 2012. Hydro-physical characterization of soils under tropical semideciduous forest. Scientia Agricola 69: 152-159.

Cunha TL. 2016. Atualização da flora arbustiva e arbórea da Estação Ecológica dos Caetetus. Undergraduate Thesis, Universidade de São Paulo, São Paulo.

Denslow JS. 1980. Gap partitioning among tropical rainforest trees. Biotropica 12: 47-55.

Denslow JS. 1987. Tropical rain forest gaps and tree species diversity. Annual Review of Ecology and Systematics 18: 431-451.

Dexter AR. 2004. Soil physical quality. Part I. Theory, effects of soil texture, density, and organic matter, and effects on root growth. Geoderma 120: 201-214.

DRYFLOR. 2016. Plant diversity patterns in neotropical dry forests and their conservation implications. Science 353: 1383-1387.

Dupuy J, Chazdon R. 2008. Interacting effects of canopy gap, understory vegetation and leaf litter on tree seedling recruitment and composition in tropical secondary forests. Forest Ecology and Management 255: 3716-3725.

Durigan G, Franco GADC, Saito M, Baitello JB. 2000. Estrutura e diversidade do componente arbóreo da floresta na Estação Ecológica dos Caetetus, Gállia, SP. Revista Brasileira de Botânica 23: 369-381.

Durigan G, Ivanauskas NM, Zakia MJB, Abreu CR. 2013. Control of invasive plants: ecological and socioeconomic criteria for the decision-making process. Natureza \& Conservação 11: 23-30.

Embrapa - Empresa Brasileira de Pesquisa Agropecuária. 1997. Manual de Métodos de Análises de Solo. 2nd. edn. Rio de Janeiro, Centro Nacional de Pesquisa de Solos.

Esquivel-Muelbert A, Baker T, Dexter K, et al. 2017. Seasonal drought limits tree species across the Neotropics. Ecography 40: 618-629. 


\section{Canopy openness and soil conditions explain community structure and diversity in a tropical seasonal forest in south-eastern Brazil}

Flora do Brasil 2020. 2018. Jardim Botânico do Rio de Janeiro. http:// floradobrasil.jbrj.gov.br. 23 Sep. 2018.

Fonseca CBR, Rodrigues RR. 2000. Análise estrutural e aspectos do mosaico sucessional de uma floresta semidecídua em Botucatu, SP. Scientia Forestalis 57: 27-43.

Gandolfi S, Joly CA, Leitão-Filho HF. 2009. Gaps of deciduousness: cyclical gaps in tropical forests. Scientia Agricola 66: 280-284.

Gandolfi S, Joly CA, Rodrigues RR. 2007. Permeability-impermeability: canopy trees as biodiversity filters. Scientia Agricola 64: 433-438.

Givnish TJ. 1988. Adaptation to sun vs. shade: a whole plant perspective. Australian Journal of Plant Physiology 15: 63-92.

Givnish TJ. 1999. On the causes of gradients in tropical tree diversity. Journal of Ecology 87: 193-210.

Givnish TJ. 2002. Adaptive significance of evergreen vs. deciduous leaves: solving the triple paradox. Silva Fennica 36: 703-743.

Gleason SM, Read J, Ares A, Metcalfe DJ. 2009. Phosphorus economics of tropical rainforest species and stands across soil contrasts in Queensland, Australia: understanding the effects of soil specialization and trait plasticity. Functional Ecology 23: 11571166.

Halpern CB, Lutz JA. 2013. Canopy closure exerts weak controls on understory dynamics: a 30-year study of overstory-understory interactions. Ecological Monographs 83: 221-237.

IBGE - Instituto Brasileiro de Geografia e Estatística. 2012. Manual técnico da vegetação Brasileira. 2nd. edn. Rio de Janeiro, IBGE.

IUCN 2018. The IUCN Red List of Threatened Species. Version 2018-1. http://www.iucnredlist.org. 05 Jul. 2018.

Ivanauskas NM, Rodrigues RR, Nave AG. 1999. Fitossociologia de um trecho de Floresta Estacional Semidecidual em Itatinga, São Paulo, Brasil. Scientia Florestalis 56: 83-99.

King DA. 1994. Influence of light level on the growth and morphology of saplings in a Panamanian forest. American Journal of Botany 81: 948-957.

Kohyama T. 1993. Size-structured tree populations in gap-dynamic forest - the forest architecture hypothesis for the strable coexistence of species. Journal of Ecology 81: 131-143.

Köppen W. 1948. Climatologia: com um estudio de los climas de la tierra. México, Fondo de Cultura Economica.

Kunstler G, Falster D, Coomes D, et al. 2016. Plant functional traits have globally consistent effects on competition. Nature 529: 204-207.

Leigh-Jr EG. 2019. Tropical seasonal forest. In: Jorgensen SE, Fath BD. (eds.) Encyclopedia of Ecology. Oxford, Elsevier. p. 684-692.

Lima RAF, Gandolfi S. 2009. Structure of the herb stratum under different light regimes in the Submontane Atlantic Rain Forest. Brazilian Journal of Biology 69: 289-296.

Lima RAF, Martini AMZ, Gandolfi S, Rodrigues RR. 2008. Repeated disturbances and canopy disturbance regime in a tropical semideciduous forest. Journal of Tropical Ecology 24: 85-93.

Lima RAF. 2005. Gap size measurement: the proposal of a new field method. Forest Ecology and Management 214: 413-419.

Linares-Palomino R, Oliveira-Filho A, Pennington RT. 2011. Neotropical seasonally dry forests: diversity, endemism and biogeography of woody plants. In: Dirzo R, Mooney H, Ceballos G, Young H (eds.) Seasonally dry tropical forests: biology and conservation. Boca Raton, Island Press. p. 3-21.

Lopes AS. 1998. Manual Internacional de Fertilidade do Solo. Piracicaba, Potafos.

Magurran AE. 2011. Medindo a diversidade biológica. Curitiba, Editora UFPR.

Martini AMZ, Lima RAF, Franco GADC, Rodrigues RR. 2008. The need for full inventories of tree modes of disturbance to improve forest dynamics comprehension: an example from a semideciduous forest in Brazil. Forest Ecology and Management 255: 1479-1488.

Melo, AS. 2008. What do we win 'confounding' species richness and evenness in a diversity index? Biota Neotropica 8: 21-27.

Metzger JP, Goldemberg R, Bernacci LC. 1998. Diversidade e estrutura de fragmentos de mata de várzea e de mata mesófila semidecídua submontana do rio Jacaré-Pepira (SP). Brazilian Journal of Botany 21: 321-330.
Molino JF, Sabatier D. 2001. Tree diversity in tropical rain forests: a validation of the intermediate disturbance hypothesis. Science 294: 1702-1704.

Moraes-Filho RM, Bonifácio-Anacleto F, Alzate-Marin AL. 2015. Fragmentation effects and genetic diversity of the key semidecidual forest species Metrodorea nigra in Southwestern Brazil. Genetics and Molecular Research 14: 3509-3524.

Morellato LPC, Rodrigues RR, Leitão-Filho HF, Joly CA. 1989. Estudo comparativo de fenologia de espécies arbóreas de florestas de altitude e mesófila semidecídua na Serra do Japi, Jundiaí, São Paulo. Brazilian Journal of Botany 12: 85-89.

Mueller-Dombois D, Ellenberg H. 1974. Aims and methods of vegetation ecology. New York, John Wiley \& Sons.

Mugasha WA, Bollandsås OM, Eid T. 2013. Relationships between diameter and height of trees in natural tropical forest in Tanzania. Southern Forests: a Journal of Forest Science 75: 221-237.

Namikawa K, Okamoto S, Sano J. 2000. Edaphic controls on mosaic structure of the mixed deciduous broadleaf/conifer forest in northern Japan. Forest Ecology and Management 127: 169-179.

Niinemets U. 2010. A review of light interception in plant stands from leaf to canopy in different plant functional types and in species with varying shade tolerance. Ecological Research 25: 693-714.

Obianga NLE, Ngomanda A, Hymas O et al. 2014. Diagnosing the demographic balance of two light-demanding tree species populations in central Africa from their diameter distribution. Forest Ecology and management 313: 55-62.

Oksanen J, Blanchet FG, Kindt R, et al. 2012. vegan-package Community Ecology Package: Ordination, Diversity and Dissimilarities. Version 2.0-5. http://cran.r-project.org/web/packages/vegan/index.html. 25 Jan. 2014.

Oliveira-Filho AT, Jarenkow JA, Rodal MJNR. 2006. Floristic relationships of seasonally dry forests of eastern South America based on tree species distribution patterns In: Pennington RT, Lewis GP, Ratter JA. (eds.) Neotropical Savannas and Dry Forests: diversity, biogeography, and conservation. Oxford, Francis CRC Press. p. 59-192.

Oliveira-Filho AT, Machado JNM. 1993. Composição florística de uma floresta semidecídua montana na Serra de São José, Tiradentes, Minas Gerais. Acta Botanica Brasilica 7: 71-88.

Oliveira-Filho AT, Scolforo JR, Mello JM. 1994. Composição florística e estrutura comunitária de um remanescente de floresta semidecídua montana em Lavras (MG). Brazilian Journal of Botany 17: 159-174.

Paciorek CJ, Condit R, Hubbell SP, Foster RB. 2000. The demographics of resprouting in tree and shrub species of a moist tropical forest. Journal of Ecology 88: 765-777.

Pérez-Harguindeguy N, Díaz S, Garnier E, Lavorel CS, et al. 2013. New handbook for standardised measurement of plant traits worldwide. Australian Journal of Botany 61: 167-234

Peters HA. 2003. Neighbour regulated mortality: the influence of positive and negative density dependence on tree populations in species rich tropical forests. Ecology Letters 6: 757-765.

Pinheiro J, Bates D, DebRoy S, Sarkar D. 2008. nlme: Linear and nonlinear mixed effects models. R Package Version 3.1-90. The R Development Core Team. http://cran.r-project.org/web/packages/nlme/index.html. 25 Jan. 2014.

Pinheiro J, Bates D. 2000. Mixed-effects models in S and S-PLUS. New York, Springer.

Pirani JR, Skorupa LA. 2002. Rutaceae. In: Wanderley MGL, Shepherd GJ, Giulietti AM. (eds.) Flora Fanerogâmica do Estado de São Paulo. 1st. edn. São Paulo, HUCITEC/FAPESP. p. 281-308.

Pires JM, Prance GT. 1977. The Amazon forest: a natural heritage to be preserved. In: Prance GT, Elias TS. (eds.) Extinction is forever. New York, New York Botanical Garden. p. 158 -194.

Pombal ECP, Morellato LPC. 2000. Differentiation of floral color and odor in two fly pollinated species of Metrodorea (Rutaceae) from Brazil. Plant Systematics and Evolution 221: 141-156.

Porder S, Vitousek PM, Chadwick OA, Chamberlain CP, Hilley GE. 2007. Uplift, erosion, and phosphorus limitation in terrestrial ecosystems. Ecosystems 10: 158-170. 


\section{Tiago Egydio Barreto, Natália Macedo Ivanauskas, Renato Augusto Ferreira de Lima, Maria Teresa Zugliani Toniato, Flaviana Maluf Souza and Ricardo Ribeiro Rodrigues}

Quesada CA, Lloyd J, Schwarz MS, et al. 2009. Regional and large-scale patterns in Amazon forest structure and function are mediated by variations in soil physical and chemical properties. Biogeosciences Discussions 5: 2003-2047.

Quesada CA, Phillips OL, Schwarz M, et al. 2012. Basin-wide variations in Amazon forest structure and function are mediated by both soils and climate. Biogeoscience 9: 2203-2012.

R Development Core Team. 2014. R: A language and environment for statistical computing. Vienna, R Foundation for Statistical Computing. http://www.R-project.org. 25 Jan. 2014.

Ramos VS, Giselda D, Franco GADC, Siqueira MF, Rodrigues RR. 2008. Árvores da Floresta Estacional Semidecidual: guia de identificação de espécies. São Paulo, EDUSP.

Rossatto DR, Carvalho FA, Haridasan M. 2015. Soil and leaf nutrient content of tree species support deciduous forests on limestone outcrops as a eutrophic ecosystem. Acta Botanica Brasilica 29: 231-238.

Runkle JR. 1982. Patterns of disturbance in some old-growth mesic forests of eastern North America. Ecology 63: 1533-1546.

Sande MT, Arets EJ, Peña-Claros M, et al. 2016. Old-growth neotropical forests are shifting in species and trait composition. Ecological Monographs 86: 228- 243.

Santos MF, Serafim H, Sano PT. 2012a. Composição e estrutura arbórea em floresta estacional semidecidual no Espinhaço Meridional (Serra do Cipó, MG). Rodriguésia 63: 985-997

Santos ML, Meira-Neto JAA, Silva AF, Martins SV, Campos EP. 2013. Estrutura fitossociológica e raridade em um trecho de Florestal Estacional Semidecidual primária na zona da mata de Minas Gerais. Global Science and Technology 06: 101-117.

Santos RM, Oliveira-Filho AT, Eisenlohr PV, Queiroz LP, Cardoso DBOS, Rodal MJN. 2012b. Identity and relationships of the Arboreal Caatinga among other floristic units of seasonally dry tropical forests (SDTFs) of north-eastern and Central Brazil. Ecology and Evolution 2: 409-428.

Scaranello MAS, Alves LF, Vieira SA, Camargo PB, Joly CA, Martinelli LA. 2012. Height-diameter relationships of tropical Atlantic moist forest trees in southeastern Brazil. Scientia Agricola 69: 26-37.

Schwarcz KD, Pataca CL, Abreu AG, et al. 2010. Genetic diversity in Atlantic Forest trees: fragmentation effects on Astronium graveolens (Anacardiaceae) and Metrodorea nigra (Rutaceae), species with distinct seed dispersal strategies. Botanical Journal of the Linnean Society164: 326-336.

Silva LA, Soares JJ. 2002. Levantamento fitossociológico em um fragmento de floresta estacional semidecídua, no município de São Carlos, SP. Acta Botanica Brasilica16: 205-216

Silva NRS, Martins SV, Meira-Neto JAA, Souza AL. 2004. Composição florística e estrutura de uma floresta estacional semidecidual montana em viçosa, MG. Revista Árvore 28: 397-405.

Silva VF, Venturin N, Oliveira-Filho AT, et al. 2003. Caracterização estrutural de um fragmento de floresta semidecídua no município de Ibituruna, MG. Cerne 9: 95-110.

Sobrado MA. 1993. Trade-off between water transport efficiency and leaf life-span in a tropical dry forest. Oecologia 96: 19-23.

Soil Survey Staff. 2014. Keys to Soil Taxonomy. 12th ed. Washington, USDA - Natural Resources Conservation Service. https://www.nrcs. usda.gov/wps/PA_NRCSConsumption/download?cid=stelprdb125 2094\&ext=pdf.
Souza FM, Gandolfi SG, Rodrigues RR. 2015. Species-specific associations between overstory and understory tree species in a semideciduous tropical forest. Acta Botanica Brasilica 29: 73-81.

Souza LA, Moscheta IS, Mourão KSM, Rosa SM. 2004. Morphology and anatomy of the flower and anthesis of Metrodorea nigra St. Hill. (Rutaceae). Brazilian Archives of Biology and Technology 47: 107-112.

Souza RP, Válio IFM. 2001. Seed size, seed germination and seedling survival of Brazilian tropical tree species differing in sucessional status. Biotropica 33: 447-457.

Souza RP, Válio IFM. 2003. Seedling growth of fifteen Brazilian tropical tree species differing in sucessional status. Brazilian Journal of Botany 26: 35-47.

Swamy V, Terborgh JW. 2010. Distance-responsive natural enemies strongly influence seedling establishment patterns of multiple species in an Amazonian rain forest. Journal of Ecology 98: 1096-1107.

Tang Z, Xu W, Zhouc G, et al. 2018. Patterns of plant carbon, nitrogen, and phosphorus concentration in relation to productivity in China's terrestrial ecosystems. Proceedings of the National Academy of Sciences 115: 4033-4038.

Turner BL, Brenes-Arguedas T, Condit R. 2018. Pervasive phosphorus limitation of tree species but not communities in tropical forests. Nature 555: 367-370.

van Lier QJ. 2014. Revisiting the S-index for soil physical quality and its use in Brazil. Revista Brasileira de Ciência do Solo 38: 1-10.

Vargas GG, Werden LK, Powers JS. 2015. Explaining legume success in tropical dry forests based on seed germination niches: A new hypothesis. Biotropica 47: 277-280.

Vico G, Dralle D, Feng X, Thompson S, Manzoni S. 2017. How competitive is drought deciduousness in tropical forests? A combined eco-hydrological and eco-evolutionary approach. Environmental Research Letters 12: 065006. doi: 10.1088/1748-9326/aa6f1b

Vidal-Torrado P, Lepsch IF, Miguel Cooper M, Gomes FH, Silva AC. 2021. Solos. In: Rodrigues RR, Ivanauskas NM. (eds.) Florestas do Estado de São Paulo: uma experiência multidisciplinar em 40 ha de parcelas permanentes. São Paulo, EDUSP (in press).

Vitousek PM, Porder S, Houlton BZ, Chadwick OA. 2010. Terrestrial phosphorus limitation: Mechanisms, implications, and nitrogenphosphorus interactions. Ecological Application 20: 5-15.

Walters MB, Reich PB. 1999. Low-light carbon balance and shade tolerance in the seedlings of woody plants: do winter deciduous and broadleaved evergreen species differ? New Phytologist 143:143-154.

Whitmore TC. 1989. Canopy gaps and the two major groups of forest trees. Ecology 70: 536-538.

Whitmore TC. 1996. A review of some aspects of tropical rain forest seedling ecology with suggestion for further enquiry. In: Swaine MD. (ed.) The ecology of tropical forest tree seedlings. Paris, Unesco. p. 3-39.

Wright J. 2002. Plant diversity in tropical forests: a review of mechanisms of species coexistence. Oecologia 130: 1-14.

Zeileis A, Kleiber C, Jackman S. 2008. Regression models for count data in R. Journal of Statistical Software 27: 1-25.

Zimmerman BL, Kormos CF. 2012. Prospects for sustainable logging in tropical forests. BioScience 62: 479-487.

Zuur AF, Ieno EN, Walker NJ, Saveliev AA, Smith GM. 2009. Mixed effects models and extensions in Ecology with R. Statistics for Biology and Health series. New York, Springer. 